
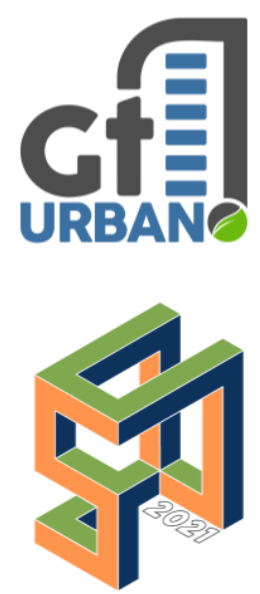

SINGEURB

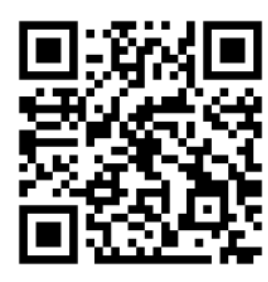

Como citar:

MORENO, Larissa

Gabriela Miras;

UEDA, Ana Cláudia.

Degradação ambiental

produzida por

resíduos de construção

civil na cidade de

Apucarana - PR. In: III

SIMPÓSIO

NACIONAL DE

GESTÃO E

ENGENHARIA

URBANA:

SINGEURB, 2021,

Maceió. Anais... Porto

Alegre: ANTAC, 2021.

p. 261-269.

Disponível em:

https://eventos.antac.o

rg.br/index.php/singe

urb/issue/view/14

\title{
Degradação ambiental produzida por resíduos de construção civil na cidade de Apucarana - PR
}

\section{Environmental degradation produced by civil construction waste in the city of Apucarana - PR}

Larissa Gabriela Miras Moreno, Universidade Tecnológica Federal do Paraná, larissamoreno@alunos.utfpr.edu.br

Ana Cláudia Ueda, Universidade Tecnológica Federal do Paraná, anaueda@professores.utfpr.edu.br

\section{RESUMO}

A construção civil é um dos setores que impacta diretamente ao ecossistema, perante aos grandes volumes de resíduos gerados que, muitas vezes, são destinados de forma inadequada, tornando-se uma problemática ambiental. Dessa maneira, o objetivo deste trabalho foi avaliar os impactos ambientais gerados pela indústria da construção civil na cidade de Apucarana - PR, por meio de uma discussão teórica e por estudo de caso de caráter exploratório, levantando dados sobre o que está sendo gerado na cidade, para o desenvolvimento da dissertação de mestrado, e até o momento, os resultados são parciais. A metodologia adotada foi por meio do método analítico quantitativo e uma pesquisa qualitativa, avaliando os dados apresentados nos Planos de Gerenciamento dos Resíduos da Construção Civil do município de Apucarana, a fim de identificar e classificar os resíduos. Diante dos elementos coletados nos 614 PGRCC, apresentados no ano de 2020, é possível identificar o grande volume gerado de resíduos de classe A e B, como também, foi identificado apenas uma empresa licenciada para disposição final dos resíduos e que atenderam no último ano a quantidade de 14.113 unidades de caçambas, e percorrendo pelos bairros do município, é possível identificar o descarte irregular em novos loteamentos. Espera-se com esse estudo, aplicar medidas e ações com conceitos sustentáveis, a fim de minimizar os danos de degradação, através de melhorias e combatendo os desperdícios.

Palavras-chave: Destinação, Impactos, Sustentabilidade.

\section{ABSTRACT}

Civil construction is one of the sectors that directly impact the ecosystem, given the large volumes of solid waste generated that are often inadequately disposed, becoming an environmental issue. Thus, the objective of this work was to evaluate the environmental impacts generated by the civil construction industry in the city of Apucarana - PR, through a theoretical discussion and exploratory case study, raising data on what is being generated in the city, for the development of the master's thesis, and so far, the results are partial. The methodology adopted was quantitative analytical method and a qualitative research, evaluating the data presented in the Civil Construction Waste Management Plans of the 
municipality of Apucarana, in order to identify and classify the waste. In view of the elements collected in the 614 PGRCC, presented in 2020, it is possible to identify the large volume generated of class $A$ and $B$ waste, as well as, only one company licensed for the final disposal of waste was identified and which served in the last year the amount of 14,113 bucket units, and walking through the districts of the municipality, it is possible to identify irregular disposal in new subdivisions. It is expected with this study, apply and action sustainable concepts, in order to minimize damage from degradation, through improvement and combating waste.

Keywords: Destination, Impacts, Sustainability.

\section{INTRODUÇÃO}

A indústria da construção civil é de extrema importância para a economia brasileira. Segundo o Instituto Brasileiro de Geografia e Estatística (IBGE), no ano de 2019 o crescimento foi de 1,6\%, chegando a R\$ 7,257 trilhões e foram contratados mais de 124 mil empregados com carteira assinada no setor. Porém, no ano de 2020 houve uma queda 5,7\%, originada pela pandemia do COVID-19 e ainda assim, foram registradas 60 mil novas vagas de emprego com carteira assinada.

Diante dos fatores positivos que se destaca no âmbito nacional, também apresentam fatores negativos. Para PASCHOALIN FILHO et al. (2014), são numerosos os sinais dos impactos ao meio ambiente gerados pelo ser humano. Com o ritmo do crescimento econômico, têm resultado no aumento significativo de resíduos sólidos urbanos e grande parte é originada pelas atividades da construção civil.

Quando se trata de questões relativas ao planejamento urbano, sucedem em degradação de ambientes naturais, instigados por construções significativas, como de indústrias, estradas e aeroportos (OLIVEIRA et al., 2014). Existe uma tendência crescente no padrão da geração de resíduos sólidos nos grandes centros urbanos, em gerar cada vez mais, afetando diretamente no meio ambiente e nos aspectos sociais e econômicos (BESSA et al., 2019).

A construção civil é a principal consumidora dos recursos naturais, conforme os materiais são extraídos, os resíduos começam a ser gerados. A formação das áreas degradadas sucede em três fases do método construtivo: na aquisição de materiais, na execução das obras civis e na disposição final dos resíduos (LARUCCIA, 2014).

Levando-se em consideração os resíduos de construção civil, é primordial não apenas a redução da geração dos resíduos, mas optar por outras opções de destinação, como a reutilização, de forma que se favoreça a formação de uma nova cadeia produtiva, transformando o resíduo em matéria-prima e contribuindo para criação de novos empregos (PASCHOALIN FILHO et al., 2014).

Para que ocorra uma destinação ambientalmente apropriada dos resíduos, se faz necessária a elaboração do plano de gerenciamento, consistindo em medidas no processo de coleta, transporte e disposição final (BESSA et al., 2019).

Nos municípios que fizeram a implantação do plano diretor para um melhor planejamento no desenvolvimento urbano, parâmetros quanto ao manejo da destinação dos resíduos estão inclusas. A 
secretária do meio ambiente é responsável pelas normas relativas ao plano de gerenciamento dos resíduos da construção civil (PGRCC) e também por sua fiscalização no cumprimento.

Em virtude do cenário desenvolvido pela indústria da construção civil, são ocasionados vários prejuízos ambientais com os altos volumes produzidos diariamente de resíduos. A consciência ecológica é de suma importância para o atual momento, os problemas ambientais devem ser encarados de forma coletiva, para que se possa alcançar o desenvolvimento de tecnologias autossustentáveis.

Neste contexto, o presente estudo tem como objetivo geral avaliar os impactos ambientais gerados pela indústria da construção civil na cidade de Apucarana - PR. Em decorrência disto, perfazem os objetivos específicos: realizar uma análise quantitativa e qualitativa na geração dos resíduos de construção civil; identificar as áreas licenciadas para disposição dos resíduos de construção civil; detectar as áreas que apresentem sintomas de degradação; propor ações que impliquem na melhoria da gestão dos resíduos de construção civil no município.

\section{MATERIAL E MÉTODOS}

A metodologia adotada foi por meio do estudo teórico: composto por revisão bibliográfica, artigos científicos nacionais e internacionais, dados estatísticos e documentos normativos e legislativos referentes aos resíduos gerados pela indústria da construção civil; e por estudo de caso por caráter exploratório: permitindo a organização e a discussão de dados levantados, a aplicação do método analítico quantitativo para determinar a classificação dos componentes de resíduos sólidos apresentados, e pela pesquisa qualitativa em relação aos problemas ambientais existentes, para que se possam projetar futuras soluções dos mesmos.

Para isto, foram avaliados os dados apresentados nos Planos de Gerenciamento dos Resíduos da Construção Civil à Prefeitura Municipal de Apucarana, a fim de identificar e classificar os resíduos. Posteriormente, atribui-se o mapeamento das áreas para disposição dos resíduos, como também, foram detectadas as áreas com despejos ilegais.

\section{1 Área de estudo}

O município de Apucarana ( $23^{\circ} 33^{\prime} 03^{\prime \prime}$ S, 51 $27^{\circ} 39^{\prime \prime}$ O) foi fundado em 28 de janeiro de 1944, está localizado no centro-norte do estado do Paraná, no Brasil. Distante a $377 \mathrm{~km}$ da capital do estado, Curitiba, com área territorial de 558,389 km² e com população estimada de 136.234 habitantes (IBGE, 2020). 
Figura 1 - Demarcação do município de Apucarana - PR.

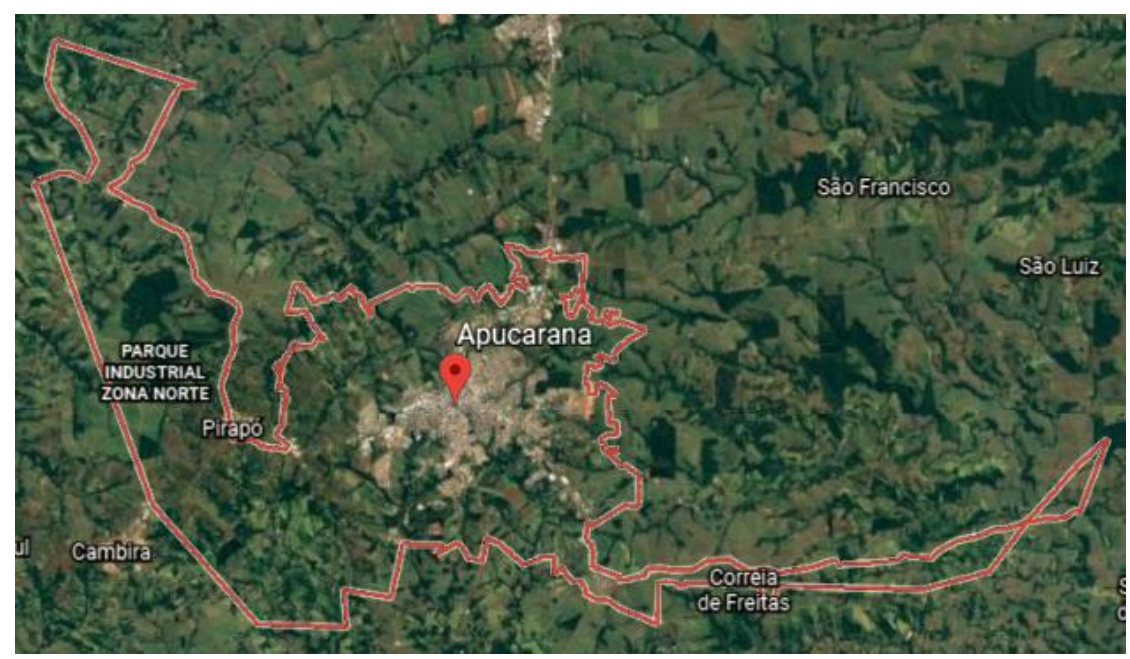

Fonte: Google Earth (2021).

\subsection{Plano de Gerenciamento dos Resíduos da Construção Civil (PGRCC)}

O Plano Integrado de Gerenciamento dos Resíduos da Construção Civil no município de Apucarana foi aprovado pela Lei ${ }^{\circ}$ 46/2014 em 06 de maio de 2014, definindo diretrizes, critérios e procedimentos para a destinação dos resíduos da construção civil, de forma que minimizem os impactos ambientais no município. Porém, apenas em 31 de julho de 2015 começou a vigorar a lei.

A finalidade do Plano de Gerenciamento dos Resíduos da Construção Civil é de classificar através de triagem os tipos de resíduos em A, B, C e D, e deverão ser destinados conforme a classificação específica.

A Resolução nº 307/2002 publicada pelo Conselho Nacional do Meio Ambiente (CONAMA), foi a primeira determinação federal imposta a respeito dos resíduos de construção e demolição, um recurso legal que determinou prazos para as administrações municipais elaborarem e implantarem planos de responsabilidade, incluindo as etapas de caracterização, triagem, acondicionamento, transporte e destinação dos resíduos. As etapas de caracterização, triagem e acondicionamento devem ser realizadas pelo grande gerador, e as etapas de transporte e destinação devem ser realizadas pelo município, diretamente ou indiretamente (pelo setor privado), remuneradas por meio de taxa.

Ao decorrer dos anos, a resolução recebeu adaptações conforme ao momento vivenciado pela sociedade. O Quadro 1 apresenta a atual classificação dos resíduos imposta pelo Conselho. 
Quadro 1 - Classificação dos resíduos da construção civil.

\begin{tabular}{|c|c|}
\hline Classe & Origem \\
\hline A & São os resíduos reutilizáveis ou recicláveis como agregados. \\
\hline B & São os resíduos recicláveis para outras destinações. \\
\hline C & $\begin{array}{l}\text { São os resíduos para os quais não foram desenvolvidas tecnologias ou } \\
\text { aplicações economicamente viáveis que permitam a sua reciclagem ou } \\
\text { recuperação. }\end{array}$ \\
\hline D & São resíduos perigosos oriundos do processo de construção. \\
\hline
\end{tabular}

Fonte: Resolução CONAMA no 307, alterada pelas Resoluções nº 348/2004, nº 431/2011 e nº 469/2015 adaptadas.

\section{RESULTADOS E DISCUSSÃO}

A partir dos elementos coletados nos 614 PGRCC, apresentados no período de 03 de janeiro de 2020 a 30 de dezembro de 2020, foi possível identificar a quantidade de resíduos por classificação, conforme exposto na Tabela 1.

Tabela 1 - Resíduos gerados no período de janeiro a dezembro do ano de 2020.

\begin{tabular}{cc}
\hline Classe & Quantidade $\left(\mathbf{k g} / \mathbf{m}^{3}\right)$ \\
\hline A & 650 \\
B & 561,46 \\
C & 35,46 \\
D & 135,10
\end{tabular}

Fonte: Secretaria do Meio Ambiente de Apucarana, 2021.

Perante aos elementos coletados, é notável a grande quantidade gerada de resíduos trituráveis, de classe A; em seguida, de classe $\mathrm{B}$, classificado como reciclável; consecutivo o de classe $\mathrm{D}$, os resíduos perigosos; e o de classe $C$, não passível de reciclagem.

Atualmente o município possui apenas uma empresa licenciada para disposição final dos resíduos de construção civil, localizada na Rua Silício, zona norte do município. 
Figura 2 - Localização da área licenciada em Apucarana - PR.

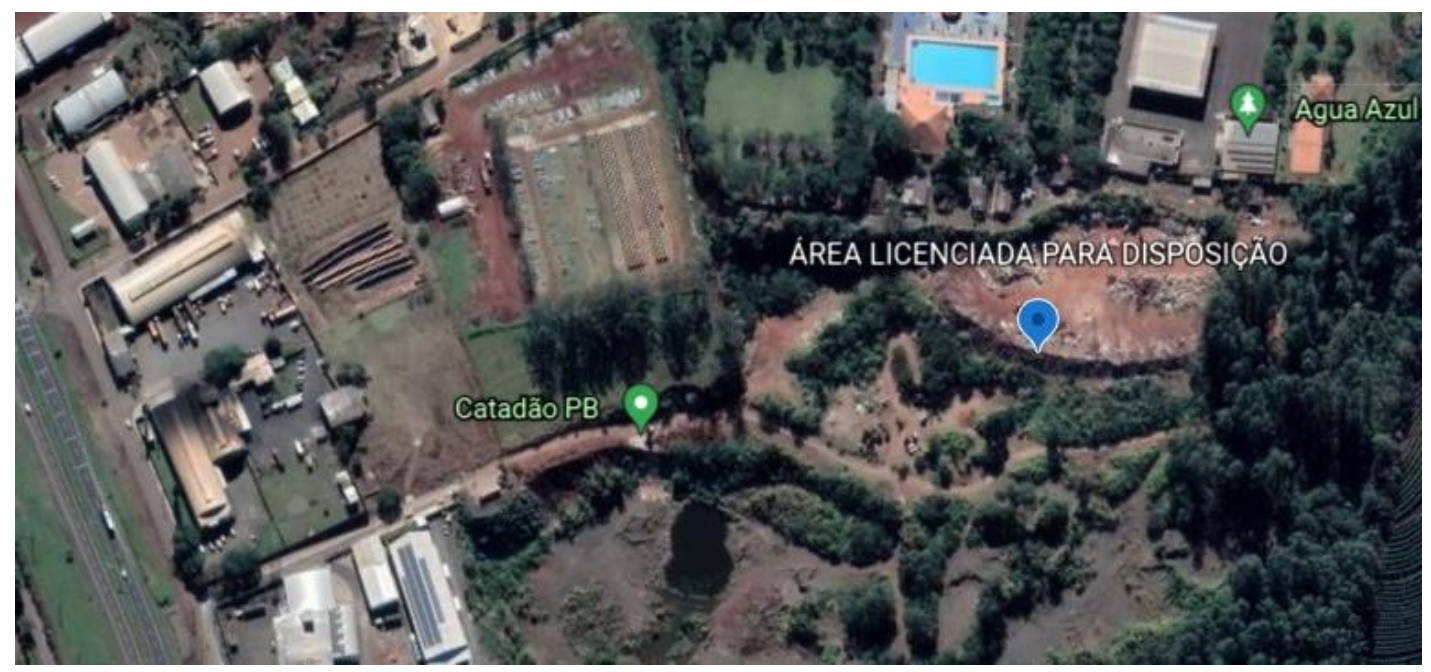

Fonte: Google Earth (2021).

A empresa licenciada em questão, informou que no ano de 2020 foram atendidas o total de 14.113 unidades de caçambas, conforme apresentado no Gráfico 1.

Gráfico 1 - Total de caçambas geradas por mês no ano de 2020.

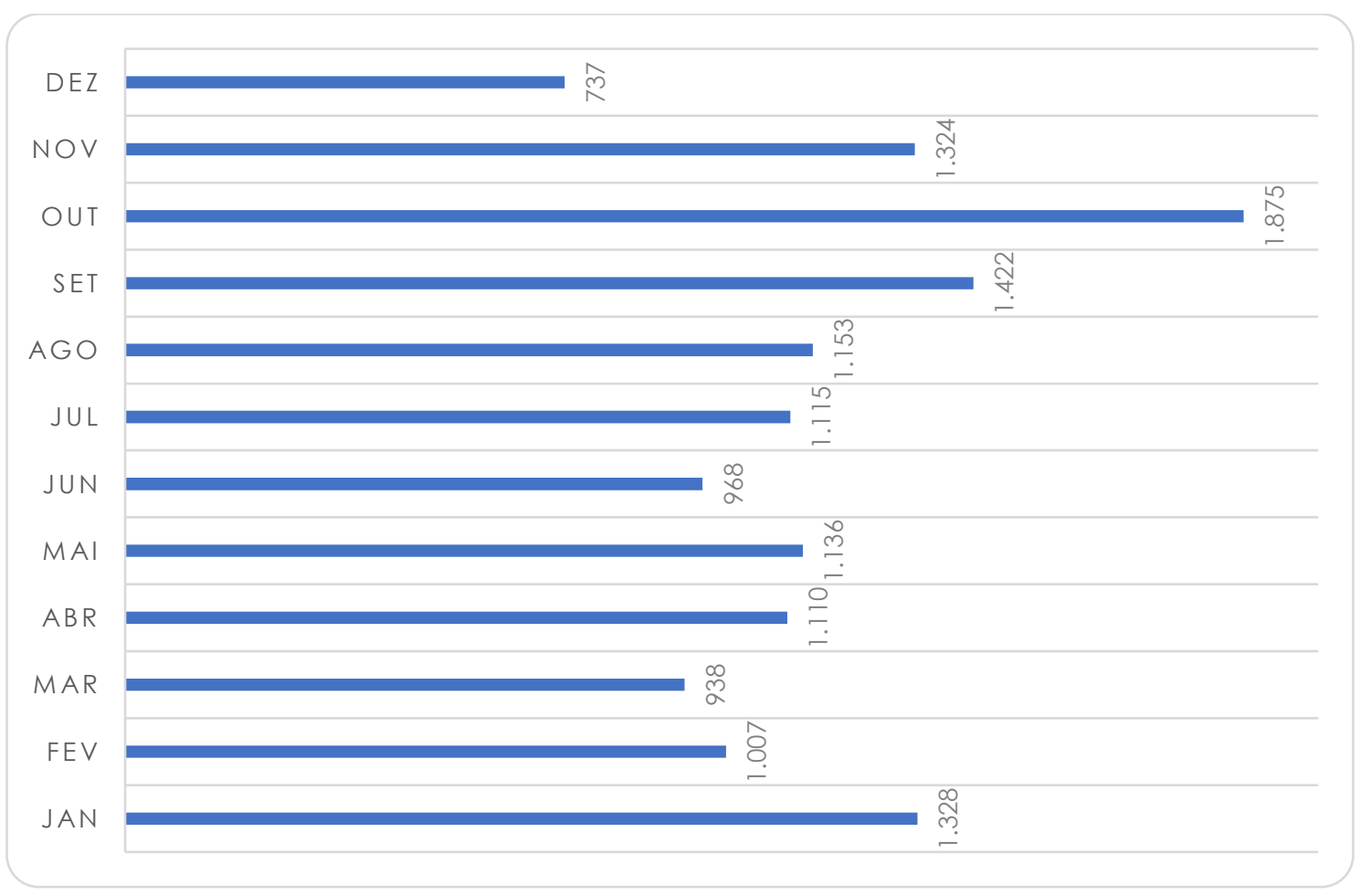

Fonte: Empresa de Apucarana, 2021. 
Diante dos dados apresentados no Gráfico 1, é evidente a oscilação nos meses de janeiro a novembro, variando de 938 a 1.875 unidades de caçambas. Contudo, o mês de dezembro é o menor, com 737 unidades de caçambas, porém, é um mês menos trabalhado devido as comemorações de final de ano.

A Prefeitura do Município de Apucarana estruturou em janeiro de 2021 um serviço de coleta de móveis e materiais inseríveis, com isso, abaixou de forma significativa o descarte irregular em fundo de vale e lotes vazios. Mas ainda assim é possível encontrar resíduo de construção civil em lotes vazios, conforme apresentado na Figura 3.

Figura 3 - Descarte irregular de RCC em Apucarana - PR.

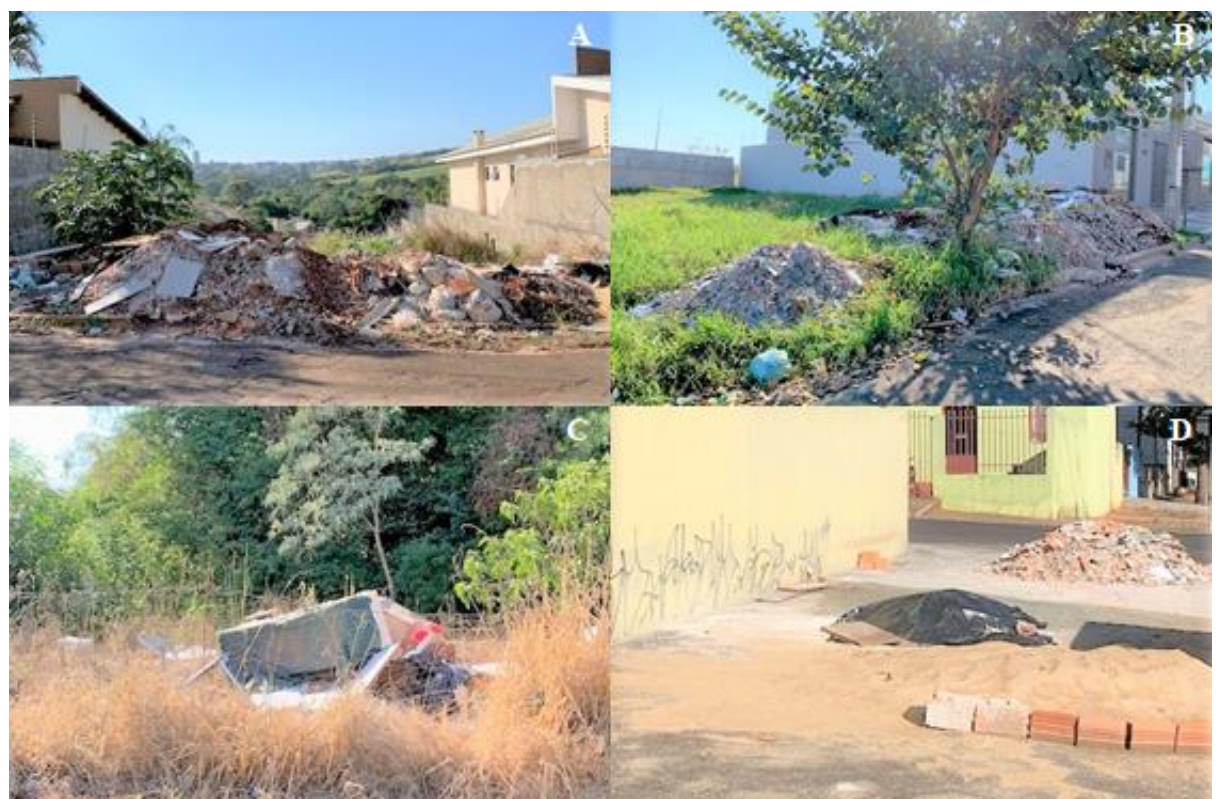

(A) Descarte irregular em lote vazio no Jardim Menegazzo; (B) Descarte irregular em lote vazio no Jardim Privamera;

(C) Descarte irregular no fundo de vale no Jardim Primavera; (D) Descarte irregular no passeio público no Núcleo

Habitacional Papa João Paulo I. Fonte: Autora, 2021.

A destinação incorreta dos resíduos tem como consequência a degradação e a poluição do solo, aumento de enchestes, proliferação de agentes transmissores de doenças, entre outros (KLEIN; GONÇALVES-DIAS, 2016). É primordial não apenas a redução da geração dos resíduos, mas optar por outras opções de destinação (PASCHOALIN FILHO et al., 2014).

Os primeiros registros sobre a reciclagem dos resíduos de construção civil no Brasil são do ano de 1997 e após a mais de 20 anos, ainda ocorrem as perdas de materiais de construção nas obras, relacionados por inúmeros fatores organizacionais, pois em cada etapa da obra acontecem as perdas e desperdícios de materiais (LIMA; LIMA, 2010).

A Resolução CONAMA nº 307/2002 remete aos municípios a elaboração e implantação de um programa de gerenciamento para esses resíduos, de forma que se reduza, reutilize e recicle, em todas as fases da obra: limpeza do terreno, montagem do canteiro, fundação, superestrutura, alvenaria, instalações hidrossanitários e elétricas, reboco, revestimentos, forro, pintura e cobertura (LIMA; LIMA, 2010). 


\section{CONCLUSÕES}

A geração de resíduos sólidos é uma das problemáticas do século XXI e as práticas exercidas pela indústria da construção civil alteram as condições ambientais, desde o processo de extração de matéria prima, no processo de construção, na edificação já finalizada e na disposição final dos resíduos, consequentemente, formando-se a áreas degradadas.

A aplicação da consciência ecológica deve ser empregada nas atividades do setor, de forma que o processo de urbanização deixe de estar diretamente ligado aos aumentos dos impactos ambientais. As aplicações do conceito de sustentabilidade devem estar empregadas em todos os processos da construção, até que se alcance um equilíbrio com o meio.

Contudo, a mudança de postura irá minimizar o consumo de recursos naturais, pois estará direcionada para reutilização, o uso de recurso renováveis e a reciclagem, mas para que as medidas ocorram, a fiscalização dos órgãos públicos como também, o conhecimento do originador sobre o que está se gerando, é de suma importância para o funcionamento de toda a cadeia produtiva.

\section{REFERÊNCIAS}

BESSA, S.A.L., et al. Quantitative and qualitative analysis of the construction and demolition waste generated in Belo Horizonte/MG. Revista Brasileira de Gestão Urbana, vol. 11, São Paulo, 2019. Disponível em: <https://www.scielo.br/scielo.php?pid=S2175-33692019000100248\&script=sci_abstract $>$. Acesso em: 17 junho 2020.

CONAMA - Conselho Nacional do Meio Ambiente. Resolução nº 307, de 05 de julho de 2002. Estabelece diretrizes, critérios e procedimentos para a gestão dos resíduos da construção civil. Diário Oficial da União, Brasília, DF. Disponível em: <http://www2.mma.gov.br/port/conama/legiabre.cfm?codlegi=307>. Acesso em: 11 junho 2020.

- Conselho Nacional do Meio Ambiente. Resolução no 348, de 16 de agosto de 2004. Altera a Resolução CONAMA no 307, de 5 de julho de 2002, incluindo o amianto na classe de resíduos perigosos. Diário Oficial da União, Brasília, DF. Disponível em: $<$ http://www2.mma.gov.br/port/conama/legiabre.cfm?codlegi=449>. Acesso em: 11 junho 2020.

- Conselho Nacional do Meio Ambiente. Resolução no 431, de 25 de maio de 2011. Altera o art. 3º da $^{\circ}$ Resolução no 307, de 5 de julho de 2002, do Conselho Nacional do Meio Ambiente - CONAMA, estabelecendo nova classificação para o gesso. Diário Oficial da União, Brasília, DF. Disponível em: $<$ http://www2.mma.gov.br/port/conama/legiabre.cfm?codlegi=649>. Acesso em: 11 junho 2020.

- Conselho Nacional do Meio Ambiente. Resolução no 469, de 29 de julho de 2015. Altera a Resolução CONAMA no 307, de 05 de julho de 2002, que estabelece diretrizes, critérios e procedimentos para a gestão dos resíduos da construção civil. Diário Oficial da União, Brasília, DF. Disponível em: $<$ http://www2.mma.gov.br/port/conama/legiabre.cfm?codlegi=714>. Acesso em: 11 junho 2020.

IBGE. Instituto Brasileiro de Geografia e Estatística. 2019. Disponível em: <https://www.ibge.gov.br/?id_noticia=1226\&id_pagina=1>. Acesso em: 31 maio 2020. 
IBGE. Instituto Brasileiro de Geografia e Estatística. 2020. Disponível em: $<$ https://www.ibge.gov.br/?id_noticia=1226\&id_pagina=1>. Acesso em: 17 julho 2021.

KLEIN, F.B., GONÇALVES-DIAS, S.L.F. A deposição irregular de resíduos da construção civil no município de São Paulo: um estudo a partir dos instrumentos de políticas públicas ambientais. Universidade Federal do Paraná, 2016. Disponível em: <https://revistas.ufpr.br/made/article/view/47703/32121>. Acesso em: 06 junho 2020.

LARUCCIA, M.M. Sustentabilidade e impactos ambientais da construção civil. Pontifícia Universidade Católica de São Paulo, 2014. Disponível em: $<$ https://ojs.eniac.com.br/index.php/EniacPesquisa/article/view/124>. Acesso em: 13 junho 2020.

LIMA, R.S., LIMA, R.R.R. Guia para elaboração de projeto de gerenciamento de resíduos da construção civil. Série de Publicações Temáticas do CREA-PR, 2010.

OLIVEIRA, D.G., et al. Land use and its impacts on the water quality of the Cachoeirinha Invernada Watershed, Guarulhos (SP). Revista Ambiente \& Água, vol. 13, n. 1, Taubaté, 2018. Disponível em: $<$ https://www.scielo.br/scielo.php?script=sci_arttext\&pid=S1980-993X2018000100301 >. Acesso em: 16 junho 2020.

PASCHOALIN FILHO, J.A., et al. Aspectos normativos a respeito de resíduos de construção civil: uma pesquisa exploratória da situação no Brasil e em Portugal. Universidade Federal do Paraná, 2014. Disponível em: <https://revistas.ufpr.br/made/article/view/32851/22443>. Acesso em: 31 maio 2020. 